\title{
Jax Info
}

National Cancer Institute

\section{Source}

National Cancer Institute. Jax Info. NCI Thesaurus. Code C41101.

Identification number (stock number) of strain at the Jackson Laboratory. 